Artículo científico

Volumen 32(1): 163-177. Enero-abril, 2021

e-ISSN 2215-3608, doi:10.15517/am.v32i1.39697

https://www.revistas.ucr.ac.cr/index.php/agromeso

\title{
Poblaciones de fitonematodos asociados al vigor de plantas de plátano ${ }^{1}$
}

\section{Populations of phytonematodes associated to the vigor of plantain plants}

\author{
Hebandreyna González-García ${ }^{2}$, Ana F. González-Pedraza ${ }^{3}$,Maryori Pineda-Zambrano ${ }^{4}$, Mery Casanova-Yepez, $^{5}$, \\ Gustavo Rodríguez-Yzquierdo ${ }^{6}$, Aníbal Soto-Bracho ${ }^{7}$
}

1 Recepción: 18 de noviembre, 2019. Aceptación: 8 de junio, 2020. Este trabajo de investigación es producto de la tesis doctoral de la primera autora, denominada: Índice de calidad en suelos cultivados con plátano (Musa AAB subgrupo plátano cv. Hartón) en el eje central de producción del sur del lago de Maracaibo, Venezuela.

2 Universidad Nacional Experimental Sur del Lago “Jesús María Semprum” (UNESUR). Programa de Ingeniería de la Producción Agropecuaria. Laboratorio de Suelos. Santa Bárbara, estado Zulia. Venezuela. gonzalezh@unesur.edu.ve (autor para la correspondencia) (https://orcid. org/0000-0001-9622-1139).

3 Universidad de Pamplona. Facultad de Ciencias Agrarias, Grupo de Investigación en Agricultura y Ganadería Sostenibles (GIAS). Pamplona, Norte de Santander, Colombia. anagonzalez11@gmail.com (https://orcid.org/0000-0002-4392-3724).

4 Universidad Nacional Experimental Sur del Lago "Jesús María Semprum" (UNESUR). Programa de Ingeniería de la Producción Agropecuaria. Laboratorio de Fitopatología. Santa Bárbara, estado Zulia. Venezuela. pinedamc@unesur.edu.ve (https://orcid.org/0000-0003-45972420).

5 Universidad Centroccidental Lizandro Alvarado. Postgrado de Agronomía. Laboratorio de Nematología. Cabudare, estado Lara. Venezuela. merycasanova@ucla.edu.ve (https://orcid.org/0000-0002-3385-9538).

6 Corporación Colombiana de Investigación Agropecuaria (AGROSAVIA). Departamento de Semillas. Sede Central, Mosquera, Cundinamarca, Colombia. grodriguezy@agrosavia.co (https://orcid.org/0000-0003-3709-8534).

7 Universidad Nacional Experimental Sur del Lago "Jesús María Semprum" (UNESUR). Programa de Administración de Empresas Agropecuarias. Santa Bárbara, estado Zulia. Venezuela. sotob@unesur.edu.ve (https://orcid.org/0000-0002-5627-5885).

\section{Resumen}

Introducción. La fracción biótica del suelo es el componente esencial en los procesos ecológicos y pueden ejercer influencia en la productividad agrícola de los cultivos. Objetivo. Identificar la población de fitonematodos asociados al vigor de las plantas de plátano en la zona de sur del lago de Maracaibo, Venezuela. Materiales y métodos. Se seleccionaron ocho lotes de plantas de plátano (Musa AAB cv. Hartón) de 2 ha, ubicados en el sur del lago de Maracaibo, los cuales se dividieron en dos áreas o lotes de 1 ha, durante octubre y diciembre del año 2018. La designación de los lotes por vigor ( $\mathrm{AV}=$ alto vigor y $\mathrm{BV}=$ bajo vigor $)$ se realizó con base en los parámetros del número de manos por racimo, circunferencia de la planta madre y altura del hijo de sucesión, en veinte plantas próximas a la cosecha, por cada uno. La muestra de suelo se recolectó a $15 \mathrm{~cm}$ de profundidad en tres plantas recién florecidas por lote y para las raíces se realizó una microcalicata por planta hasta $30 \mathrm{~cm}$ de profundidad. Para la extracción nematológica en suelo se empleó el método de levigación de oostenbrink con embudo de Baermann y en raíz el método de licuado. Posteriormente, se cuantificó por género los especímenes encontrados. Resultados. Se encontró una población en el suelo (14 140 individuos) más significativa ( $<<0,05)$ que en la raíz (2670 individuos). Al comparar la población total por vigor no se observó diferencia estadística entre AV (395) y BV (305). Se determinaron doce géneros taxonómicos en las muestras de raíz y en suelo. Los especímenes más predominantes en AV fueron Helicotylenchus multicinctus y Radopholus similis y en los lotes BV fue Pratylenchus sp. Conclusión. Se observó una alta diversidad de la nematofauna presente tanto en los suelos como en raíces del cultivo.

Palabras claves: clon de plátano Hartón, nematodos, productividad agrícola.

(C) 2020 Agronomía Mesoamericana es desarrollada en la Universidad de Costa Rica bajo una licencia Creative Commons Atribución-NoComercial-SinDerivar 4.0 Internacional. Para más información escriba a pccmca@ucr.ac.cropccmca@gmail.com 


\begin{abstract}
Introduction. The biotic fraction of the soil is the essential component in ecological processes and can influence agricultural productivity of crops. Objective. To Identify the population of phytonematodes associated with plantain plants vigor in the southern zone of lake Maracaibo, Venezuela. Materials and methods. Eight 2-hectare batches of plantain plants (Musa AAB cv. Hartón), located in the south of lake Maracaibo, were selected and divided into two 1-hectare areas or lots, during October and December of the year 2018. The designation of the lots by vigor (HV= high vigor and $\mathrm{BV}=$ low vigor) was made based on the parameters of the number of hands per cluster, circumference of the mother plant, and the height of the son of succession, in twenty plants close to harvest, for each one. The soil sample was collected at a depth of $15 \mathrm{~cm}$ on three newly flowered plants per lot and for the roots a microcalicata was made per plant up to $30 \mathrm{~cm}$ deep. For the nematological extraction in the soil, the oostenbrink levigation method was used with a Baermann funnel and the blending method was used as a root. Subsequently, the specimens found were quantified by gender. Results. A population more significant $(\mathrm{p}<0,05)$ than in the root $(2670$ individuals) was found in the soil (14 140 individuals). No statistical difference between AV (395) and BV (305) was observed when comparing the total population by vigor. Twelve taxonomic genera were determined in the root and soil samples. The most prevalent specimens in AV were Helicotylenchus multicinctus and Radopholus similis and in the BV lots it was Pratylenchus sp.
\end{abstract} Conclusion. A high diversity of nematofauna present in both soils and roots of the crop was observed.

Keywords: Harton banana clon, nematodes, agricultural productivity.

\title{
Introducción
}

El plátano es el cuarto cultivo más importante del mundo después del arroz, el trigo y el maíz (Food and Agriculture Organization, 2004). Esta fruta constituye la base de la alimentación de muchas regiones tropicales y, además representa una importante fuente de ingresos para los productores. Aproximadamente el $70 \%$ de la producción nacional de plátano se concentra en la zona Sur del Lago de Maracaibo, específicamente en los estados Zulia, Trujillo, Mérida y Táchira. El estado Zulia, por su parte, ocupa el mayor número de áreas sembradas y las más grandes unidades de producción, especialmente aquellas situadas entre los ríos Escalante, Mucujepe y en las riberas del río Chama (Martínez, 2009).

La información generada sobre la morfología de la cepa del plátano Hartón y el ciclo de las plantas, está relacionada con la producción. La longitud del pseudotallo y su circunferencia están en relación directa en primer término con el tipo de clon y luego con el vigor de la planta producto de su estado de crecimiento (Soto, 2008).

El número de manos por racimo, la circunferencia del pseudotallo y la altura del hijo de sucesión, son parámetros que están altamente correlacionados con la producción o vigor de la plantación en banano o plátano (Rosales et al. 2008). Datos relacionados con la biometría de la cepa de plátano (Musa AAB subgrupo plátano cv. Hartón), en la planicie del río Chama, depresión del Lago de Maracaibo, muestran que el perímetro promedio del pseudotallo de la planta madre fue de 76,36 cm (Rodríguez \& Rodríguez, 1998); valor recomendado como base para evaluar el manejo de la plantación, complementar el diagnóstico nutricional del cultivo y determinar su potencial productivo, en el área estudiada.

En un estudio en la zona de El Paraíso, municipio Colón, al sur del lago de Maracaibo, no encontraron diferencias estadísticamente significativas en el número de manos por racimo (Hernández et al., 2007). En el mismo estudio se señaló que las respuestas de la fertilización en plátano, el número de manos y los dedos por mano, son variables poco afectadas por tratamientos de fertilización (Hernández et al., 2007). 
De igual forma, el plátano, como todos los cultivos, es susceptible de ser atacado por un grupo de nematodos que afectan su desarrollo y la producción. Las pérdidas ocasionadas por estos fitopatógenos dependen de la población existente, de la susceptibilidad del material, del tipo de suelo y las condiciones ambientales que favorezcan la infección, alimentación y reproducción de estos (Belalcázar, 1991).

En condiciones óptimas para el desarrollo de las plantas, estas absorben los nutrimentos del suelo a través de la raíz, lo que conforma una amplia red de distribución de alimentos brutos o fotoasimilados que fomentan el crecimiento, desarrollo y desempeño productivo de la planta. Al lesionar este tejido e invadirlo como lo hacen los nematodos, las plantas se ven limitadas para absorber agua y nutrientes, lo cual provoca condiciones de estrés, bien sea porque estén sometidas a estrés biótico o abiótico, y así segregan hacia el suelo mayor cantidad de exudados (Badri \& Vivanco, 2009), lo cual desempeña un papel importante en los mecanismos de atracción o repelencia química en la rizósfera, y ella activa los componentes químicos que alteran el comportamiento de los nematodos atrayéndolos hacia la raíz (Peña et al., 2012; Torres-Guerrero et al., 2013).

Los nematodos en los cultivos pueden causar problemas de anclaje y fuertes daños al sistema radical, lo que provoca lesiones y pudrición de este, lo cual afecta en gran medida el desarrollo normal de la planta (Bautista et al., 2015). Se han reportado 146 especies de parásitos o asociados al género Musa, distribuidas en 43 géneros, considerándose los más devastadores y ampliamente distribuidos: Radopholus similis y Pratylenchus coffeae (endoparásitos migratorios) y Helicotylenchus multicinctus (ectoparásito migratorio). De menor importancia se reportan las especies del género Meloidogyne (endoparásito sedentario) y Rotylenchus reniformis (semiendoparásito sedentario) (Gowen et al., 2005).

El nemátodo barrenador ( $R$. similis) es el de mayor importancia económica en la mayoría de las regiones donde se cultivan banano y plátano (Gowen y Quénéhervé, 1990; Montiel et al., 1997). Por su parte, el nemátodo espiral (H. multicinctus) es probablemente, después de $R$. similis, el más numeroso y ampliamente diseminado en las plantaciones de banano y plátano (Gowen et al., 2005; Torrado-Jaime \& Castaño-Zapata, 2009).

En cuanto a la especie Pratylenchus, se ha encontrado que, por sus características endoparasíticas, penetra por la zona cortical de las raíces y produce una lesión rojiza, la cual avanza a medida que el nemátodo se alimenta, hasta tornarse necrótica (Crozzoli, 2002). Posiblemente las sinergias establecidas con los productos aplicados para el manejo como el biofertilizante y la fertilización mineral, permiten recuperar e incrementar la biota edáfica, lo que favorece el incremento de la actividad biológica, la fijación mineral y la compleja regulación de parasitismo reduce los daños en lotes de alto vigor de plantas de plátano.

En varios sectores (Caño blanco, Moralito, Mosioco, Taparones, Caño muerto, El Uvito y el Treinta y Cinco) del sur del lago de Maracaibo, Venezuela, estos géneros H. multicinctus, $P$. coffeae, $R$. similis y Meloidogyne, se encontraron destacando algunas variaciones numérica entre sectores, razón para que fuese considerado como el nemátodo de mayor distribución en la zona (Montiel et al., 1997).

Las pocas investigaciones que se han hecho en el sur del lago de Maracaibo, Venezuela, han estado relacionadas con la cuantificación de poblaciones de nematodos en diferentes épocas de muestreo (Abou Assi et al., 2009; Labarca et al., 2011); sin embargo, no se ha abordado con mayor amplitud las poblaciones asociadas con el vigor de plantas de plátano.

El objetivo de esta investigación fue identificar la población de nematodos asociados al vigor de plantas plátano en la zona de sur del lago de Maracaibo, Venezuela. 


\section{Materiales y métodos}

\section{Descripción del área de estudio}

El estudio se realizó en el eje central de mayor producción de plátano (Musa AAB), en el sur del lago de Maracaibo, Venezuela, conformado por 35 unidades de producción, las cuales se encuentran ubicadas en los $\mathrm{km}$ 41 y 35 (sector El Uvito), sector Bancada de Limones, sector Caño Negro, sector 4 Bocas, de la parroquia el Moralito del municipio Colón del estado Zulia; ubicadas a una altitud de $8 \mathrm{msnm}$, con precipitación promedio de $2200 \mathrm{~mm}$, una temperatura de $27^{\circ} \mathrm{C}$ y una humedad relativa máxima de $90 \%$ (Gómez, et al., 2016). La época de menor precipitación para el año 2018 fue durante los meses de julio, agosto y septiembre. Los suelos están clasificados como: Typic tropopsamments mixto e isohipertémico de la serie Bancham y Fluventic Eutropepts mixto e isohipertémico de la serie Chama, con inclusiones de la serie Chamita (Kijerwski et al., 1976). El área se caracteriza por el predominio absoluto de partes planas y algunas ciénagas; en cuanto a paisaje y relieve, se trata de una planicie aluvial baja y llanura aluvial plana, respectivamente. La composición geomorfológica de esta zona presenta un predominio de napas que engloban el $54 \%$ de la superficie total y es representado por las series Chama, Padre, Raya y Oncha, las cuales presentan influencia del río onia; las demás posiciones están representadas por las cubetas de desborde de la serie Puerto con materiales de los ríos Onia y Escalante, que ocupan el $29 \%$ de la superficie, el $6 \%$ está representado por los brazos colmatados de la serie Bancham y un $10 \%$ de albardones de los brazos activos de la asociación Chama, Padre y Chamita (Kijerwski et al., 1976).

\section{Selección de los sitios de muestreo}

El proceso de selección de los sitios de muestreo se realizó entre octubre y diciembre del año 2018 y se dividió en dos etapas: la primera consistió en un prediagnóstico, el cual tuvo por finalidad obtener el historial de la finca, basado en entrevistas con los productores, para colectar información sobre condiciones de manejo y situación productiva de la finca. El historial se obtuvo a través de un diálogo con el productor con la inclusión de temas sobre el manejo agronómico y comportamiento histórico productivo de la finca, cultivares usados, población o densidad de plantación, producción por hectárea, retornos, entre otros. También se indagó sobre el manejo y la calidad del agua de riego (si existe), programa de fertilización (últimos tres años como mínimo), problemas y prácticas fitosanitarias de la finca (sigatoka, nematodos, insectos, malezas) y se colectó información disponible tales como: mapas de suelos, riego y/o drenaje, análisis de suelo y foliares (últimos tres años como mínimo) y datos climatológicos (precipitación y temperatura semanal).

La segunda etapa consistió en la identificación dentro de la finca de dos áreas o sitios de muestreo: uno con plantas de alto vigor (AV) y otro con plantas de bajo vigor (BV), según el criterio del productor. El vigor de las plantas en este estudio se obtuvo con base en los siguientes parámetros: circunferencia de la planta madre $(\mathrm{cm})$, altura del hijo de sucesión (m) y número de manos por racimo.

Para la selección del tamaño de las fincas, se tomó en cuenta el criterio recomendado por Rosales et al. (2008) para el diagnóstico de la calidad y salud de los suelos cultivados con banano, pero en este caso aplicado al plátano. Se seleccionó una muestra de siete unidades de producción de plátano (Arias, 2006) de las 35 que comprenden el eje, de las cuales, seis cuentan con una superficie de menos de 10 ha y la otra entre 12 y 15 ha. De acuerdo con la metodología propuesta por Rosales et al. (2008), en las unidades de producción con tamaños menores a 10 ha, se tomó un área de muestreo con plantas de alto vigor y otra área con plantas de bajo vigor, mientras que en fincas de hasta 25 ha, dos áreas de muestreo por cada lote de vigor. Por lo tanto, en las seis unidades de producción con menos de 10 ha se delimitó una parcela de $20 \mathrm{~m}$ por $50 \mathrm{~m}\left(1000 \mathrm{~m}^{2}\right)$ por cada lote de vigor; y en la unidad con más de 10 ha se demarcó dos áreas con la misma superficie por cada lote vigor. 


\section{Determinación del vigor de las plantas}

La selección de lotes con plantas de bajo y alto vigor se realizó tomando el criterio señalado por Rodríguez \& Rodríguez (1998), con valores de circunferencia del pseudotallo de la planta madre por encima de $76,36 \mathrm{~cm}$ en promedio y siete manos por racimo, pueden ser consideradas como el arquetipo ideal de la cepa madre de elevada productividad del plátano Hartón en Venezuela, así como también dieciséis hojas activas por retoño y una media de 5,48 hojas con ancho de lámina foliar mayor de $10 \mathrm{~cm}$. Estos autores también indicaron que la comparación de estas características biométricas de las plantas madre y su retoño, con las de una plantación cualquiera, permitirían determinar si su potencial productivo es alto, así como también recomendaron utilizar los valores antes mencionados, como base para el manejo de la plantación, complementar el diagnóstico nutricional del cultivo y determinar su potencial productivo.

Dentro de cada lote de vigor se seleccionaron veinte plantas con racimos próximos a cosecha (tres semanas), para un total de cuarenta plantas por área de alto y bajo vigor, respectivamente. En el caso de no encontrar las veinte plantas dentro de los $1000 \mathrm{~m}^{2}$, se seleccionaron plantas vecinas que cumplían los requisitos para contar con la cantidad necesaria. Se calculó la densidad poblacional mediante el conteo total de las plantas dentro del lote de $1000 \mathrm{~m}^{2}$ y multiplicando el número de plantas encontradas por 10 (Rosales et al., 2008).

Para evaluar el vigor de las plantas se procedió a medir los siguientes parámetros: número de manos por racimo, circunferencia del pseudotallo de la planta madre y altura del hijo de sucesión. El número de manos por racimo que se obtuvo contando todas las manos de cada racimo. La circunferencia de la planta madre, se evaluó al momento de la floración, cuyo valor se obtuvo midiendo la circunferencia $(\mathrm{cm})$ del pseudotallo, esta fue medida de la base del suelo hasta los $100 \mathrm{~cm}$ de altura, para ello se utilizó una cinta métrica. La altura del hijo de sucesión (m) se midió al momento de la cosecha, para lo cual se tomó en cuenta el hijo que se encuentra al frente de la planta madre. Para ello se procedió a medir desde el nivel del suelo hasta la inserción del pseudopecíolo de la última hoja expandida con la hoja candela.

\section{Determinación de nematodos en suelos}

Se eligieron tres plantas de plátano recién florecidas con un hijo de sucesión de 1,2 a 1,8 m de altura, en un solo momento (Rosales et al., 2008). A $10 \mathrm{~cm}$ frente al hijo de sucesión se realizó una excavación con el palín holandés de $15 \mathrm{~cm}$ de profundidad. Se introdujo el palín de dos a tres veces en el mismo hoyo para obtener aproximadamente $1 \mathrm{~kg}$ de suelo por punto de muestreo, el cual se depositó en una bolsa plástica. Todas las muestras se identificaron y transportaron al laboratorio en una hielera.

Posteriormente, el suelo fue cernido en el laboratorio de nematología de la Universidad Centroccidental Lisando Alvarado, Lara, Venezuela. Los nematodos se extrajeron utilizando el método de elutriador o de levigación de Oostenbrink, que permite la separación de partículas pesadas del suelo y restos de raíces mediante la acción física del proceso de levitación; para ello se colocó el tubo perforado en la parte inferior del aparato, dicho tubo controló la entrada de agua al mismo. Se abrió la llave para permitir el flujo de agua (100 $\left.\mathrm{ml} \mathrm{min}^{-1}\right)$. El tanque del elutriador o levigador se llenó de agua hasta el nivel de salida.

La muestra o sub-muestra de suelo fue de $100 \mathrm{~g}$; esta se colocó en el embudo superior y se lavó con una ducha (utilizando la mano para lavar y revolver bien la muestra durante el proceso). El lavado se realizó hasta llenar dos terceras partes de la columna del elutriador (nivel 1), momento en el cual se debió cerrar el flujo superior y dejar solo el inferior, reduciéndolo a $600 \mathrm{ml} \mathrm{min}^{-1}$ hasta que el elutriador se terminara de llenar. Cuando el elutriador estuvo el $80 \%$ lleno, se abrió el drenaje lateral de la solución, se vertió sobre un conjunto de cuatro tamices (cribas número 325) colocados en serie frente al dispositivo de salida. Las partículas y nematodos retenidos en estos tamices se trasladaron mediante lavado con agua corriente a un vaso de precipitado. 
Las muestras obtenidas por lo general fueron muy turbias, por lo que se pasaron por una fase de limpieza (Jacob \& Van-Bezooijen, 1971), con el método de embudo de Baermann, para lo cual se insertaron los trozos de tubo de goma en los cuellos de los embudos y se cerraron con pinzas, consecutivamente se llenaron los embudos con agua. Posteriormente, se colocó un trozo de malla en cada embudo hasta la mitad del cono, luego se sacó un poco de agua por el cuello con el fin de desalojar el aire. De igual manera, se colocó una hoja de papel facial sobre la malla en el embudo y se vertió el contenido del vaso precipitado. Se añadió más agua al embudo dejándola correr suavemente por las paredes, hasta que el suelo quedara ligeramente embebido en el agua (aproximadamente $1 \mathrm{~cm}$ ). Se dejó la muestra por 24 h, y se agregó agua cada vez que el nivel de esta se separó del suelo. Así, los nematodos se dirigieron hacia la humedad, atravesaron el papel facial y se ubicaron en el cuello del embudo, concentrándose por encima de la pinza. Transcurridas $24 \mathrm{~h}$, se extrajo de 5 a $10 \mathrm{ml}$ de agua en tubos de ensayos y se les agregó una porción de formol al $10 \%$, el cual se calentó a $80^{\circ} \mathrm{C}$ y se dispersó en el recipiente contentivo de la suspensión de nematodos para la muerte y fijación de estos, inmediatamente se tapó la muestra para su posterior identificación.

Se realizaron montajes de láminas permanentes por el método rápido de Baker (Jacob \& Van-Bezooijen, 1971), el cual consiste en colocar el nemátodo (juvenil de segundo estado) en láminas cavadas con una gota de lactofenol, a una temperatura entre 60 y $70{ }^{\circ} \mathrm{C}$ en baño de vapor seco durante $3 \mathrm{~h}$. Con este mismo proceso se pasó de manera consecutiva por cinco soluciones más, cada una durante 3 h (Cuadro 1).

Cuadro 1. Soluciones utilizadas para el método rápido de Baker. Maracaibo, Venezuela. 2018.

Table 1. Solutions used for Baker's quick method. Maracaibo, Venezuela. 2018.

\begin{tabular}{|c|c|c|c|c|c|c|}
\hline Baker & $\mathbf{0}$ & 1 & 2 & 3 & 4 & 5 \\
\hline Lactofenol & 100 & & & & & \\
\hline Glicerina & & 55 & 70 & 82 & 90 & 100 \\
\hline Ácido láctico & & 15 & 10 & 5 & 2,5 & \\
\hline Cristales de Fenol & & 15 & 10 & 5 & 2,5 & \\
\hline Agua destilada & & 10 & 5 & 5 & 2,5 & \\
\hline Formalina & & 5 & 5 & 3 & 2,5 & \\
\hline
\end{tabular}

Fuente / Source: Dropkin (1989).

Luego que los especímenes se pasaron por cada una de las soluciones, se procedió a la preparación de las láminas permanentes. En una lámina portaobjetos se colocó un anillo de parafina, el cual se obtuvo de calentar la boca de un tubo de cobre (1,5 cm de diámetro) con la llama de un mechero y se tocó la parafina sólida; $\sin$ dejar enfriar, se puso en contacto la boca del tubo con la lámina portaobjetos, así se dejó marcado el anillo de parafina. En el centro del anillo se colocó una gota de glicerina deshidratada y en el centro de la gota el nemátodo. Posteriormente, se ubicó sobre el anillo un cubreobjetos y luego la lámina se pasó sobre el mechero, de esta forma la parafina se derritió, quedando la gota de glicerina en el centro; y así cuando se solidificó nuevamente la parafina, la lámina quedó sellada.

Se identificaron las especies con la ayuda de claves, dibujos obtenidos de los trabajos originales y revisiones de géneros. 


\section{Determinación de nematodos en raíces}

Para determinar los nematodos en raíces se seleccionaron tres plantas recién florecidas con hijos de sucesión de 1,2 a 1,8 m de altura, una sola vez. En cada planta, a $10 \mathrm{~cm}$ frente al hijo de sucesión, se abrió una minicalicata; con una excavación de $13 \mathrm{~cm}$ de largo, $13 \mathrm{~cm}$ de ancho y $30 \mathrm{~cm}$ de profundidad, y se colectaron las raíces en bolsas plásticas bien identificadas.

Las raíces de las tres plantas se mezclaron para conformar una muestra compuesta, que fueron almacenadas a una temperatura entre 8 a $10^{\circ} \mathrm{C}$ (Rosales et al., 2008). Posteriormente, en condiciones de laboratorio, las raíces se lavaron con agua potable para remover el suelo y los residuos. Seguidamente, con un cuchillo o una tijera, se separaron las raíces funcionales y no funcionales. Raíces funcionales son aquellas de color blanco o crema, a veces con coloraciones café rojizo, pero sin tejido necrosado. Raíces no funcionales son aquellas de color negro con tejido necrosado. Luego las raíces funcionales y no funcionales se pesaron por separado. Posteriormente, los segmentos de las raíces funcionales (únicamente) se homogenizaron en una bolsa plástica agitando manualmente. De esta bolsa, se tomaron cinco grupos de $4 \mathrm{~g}$ de raíces cada uno, hasta obtener un total de $20 \mathrm{~g}$ de raíces funcionales. Se colocaron los $20 \mathrm{~g}$ de raíces funcionales en la licuadora y se agregó agua potable hasta cubrir las raíces.

Se licuaron las raíces a baja velocidad por $20 \mathrm{~s}$, luego se filtró el contenido en un tamiz de $325 \mathrm{~mm}$ (se lavó inclusive la licuadora dentro del tamiz) y se recolectó en un vaso precipitado. Sobre el vaso precipitado se colocó papel facial y sobre el montaje del papel una malla. Seguidamente, se colocó el vaso con la malla dentro del embudo de Baermann (el cual previamente se encontraba ajustando el torniquete con una pinza y agua de chorro); se dejó en reposo 48 h, luego, se recolectó el contenido en un tubo de ensayo y se le agregó una porción de formol al 10 $\%$ igual a la cantidad de suspensión de los nematodos. Dicho formol se calentó a $80{ }^{\circ} \mathrm{C}$ y se vertió en el recipiente que contenía la suspensión de nematodos, inmediatamente se tapó la muestra para su posterior identificación.

Para el montaje de láminas permanentes, así como también la fijación de las mismas con los especímenes para su identificación, se utilizó la misma metodología anteriormente descrita para nematodos en suelos. Los resultados para el caso de nematodos en suelos se expresaron en individuos $100 \mathrm{~g}^{-1}$ de suelo y para raíces individuos $20 \mathrm{~g}^{-1}$ de raíces funcionales.

\section{Diseño experimental}

El diseño experimental utilizado fue completamente al azar, para ello se procedió a realizar un análisis estadístico univariado, mediante el cual se determinaron parámetros estadísticos básicos, como la media y el error estándar, además se realizó la comparación de medias con la prueba de Tukey y una probabilidad de $\mathrm{P}<0,05$, en el programa Statistix versión 8,0 .

\section{Resultados}

\section{Descripción del manejo agronómico de las unidades de producción}

Las unidades de producción se encontraban cultivadas con el clon de plátano Musa AAB cv. Hartón (Nava, 1997); la edad de la plantación era de tres años, con un manejo perenne. El manejo cultural, correspondiente al deshoje y deshije, era similar en todas las fincas. La primera práctica se realizaba dentro del primer mes y medio, y de dos a tres meses la segunda. En cuanto al manejo de malezas, la mayor parte de las fincas realizaba un control manual (con guadaña), además se aplicaban herbicidas, ya sean de contacto o sistémico no selectivos en dosis que iban de 1 a 21 en 2001 de agua, con una frecuencia de aplicación de cada dos o tres meses. Los insecticidas 
aplicados entre fincas variaban, en dosificaciones que están entre $500 \mathrm{ml}$ y 11 por 2001 de agua, de: Strongly Lorsban, Lambda y Mercamil cada cuatro, seis o doce meses, respectivamente.

Las aplicaciones de fungicidas se hacían a razón de 1 a 21 del insumo sintético por cada 2001 de agua, con productos que iban desde propizole, carbendazin, timore o curacarb, a base de propiconazole y benzimidasole. En una de las unidades de producción (La Mano de Dios) se aplicaba propizole, timore o curacarb, mezclado con 8 1 de rocío blanco (aceite); $250 \mathrm{cc}$ de hormovita u hormoplus y $100 \mathrm{cc}$ de BioCrece (estimulante de la floración). El productor de la finca agropecuaria Dos Ríos empleaba un producto biológico foliar, denominado VenAgro, a base de quitosano, cada tres meses, con una dosis de 21 en 2001 de agua. Finalmente, los productores encuestados expresaron que los planes de fertilización iban en función de la disponibilidad del mercado y que no contaban con riego. Sin embargo, coincidieron en la aplicación de forma individual de triple 15-15-15, 10-20-20 y urea, con dosis entre 100, 200 y $300 \mathrm{~g} \mathrm{planta}^{-1}$, cada tres meses. El triple 18-18-18, lo aplicaban cada 21 días en dosis de $1 \mathrm{~kg}$ en 2001 de agua o mensualmente, $4 \mathrm{~kg}$ en 2001 de agua.

\section{Determinación de vigor de las plantas}

Se observó que el número de manos por racimo, la circunferencia de la planta madre $(\mathrm{cm})$ y la altura del hijo de sucesión $(\mathrm{m})$, fueron significativamente $(\mathrm{p}<0,05)$ más altos en lotes de plantas de plátano de alto vigor $(\mathrm{AV})$ con respecto a los de bajo vigor (BV). Asimismo, al realizar la estimación de la densidad de plantas por hectáreas se reflejó una tendencia mayor en lotes de alta producción (Cuadro 2).

Cuadro 2. Vigor de plantas de plátano (Musa AAB cv. Hartón) en fincas del sur del lago de Maracaibo, Venezuela. 2018.

Table 2. Vigor of plantain plants (Musa AAB cv. Hartón) in farms in the south of lake Maracaibo, Venezuela. 2018.

\begin{tabular}{ccccc}
\hline & \multicolumn{3}{c}{ Parámetros biométricos de plantas de plátano } \\
\hline $\begin{array}{c}\text { Lotes de vigor de } \\
\text { plantas }\end{array}$ & $\begin{array}{c}\text { Número de manos por } \\
\text { racimo }\end{array}$ & $\begin{array}{c}\text { Circunferencia de la } \\
\text { planta madre }(\mathbf{c m})\end{array}$ & $\begin{array}{c}\text { Altura del hijo de } \\
\text { sucesión }(\mathbf{m})\end{array}$ & $\begin{array}{c}\text { Densidad poblacional } \\
\left(\text { plantas ha }^{-1}\right)\end{array}$ \\
\hline Alto vigor & $7,50 \pm 0,97^{\mathrm{a}}$ & $66,86 \pm 8,29^{\mathrm{a}}$ & $2,56 \pm 0,53^{\mathrm{a}}$ & 3140 \\
Bajo vigor & $4,96 \pm 0,89 \mathrm{~b}$ & $59,44 \pm 9,16 \mathrm{~b}$ & $2,03 \pm 0,48 \mathrm{~b}$ & 2595 \\
\hline Probabilidad & $<0,05$ & $<0,05$ & $<0,05$ & \\
\hline
\end{tabular}

Valores promedios \pm desviación estándar seguidos por letras minúsculas diferentes entre las columnas indican diferencias significativas $(\mathrm{p}<0,05)$ según prueba Tukey entre lotes de plantas de plátano de alto y bajo vigor / Average values \pm standard deviation followed by different lowercase letters between the columns indicate significant differences $(p<0.05)$ according to Tukey's test between lots of high and low-vigor plantain plants.

\section{Determinación de nematodos en suelos y raíces de plantas de plátano}

Se cuantificó en total 16810 individuos. La cantidad de especímenes presentaron fluctuaciones según el lote procesado (Figura 1).

La población cuantificada por lotes de plantas de plátano estudiados (AV y BV) presentó una respuesta divergente entre la cantidad de individuos contados y el tipo de muestra estudiada (Figura 2). No existió diferencia estadística entre los lotes de plantas de plátano en la cantidad de nematodos presente en muestras de suelo y en las poblaciones totales. Sin embargo, se observó una tendencia de mayor población de nematodos fitopatógenos en el 
$\square$ Alto Vigor $\quad \square$ Bajo Vigor

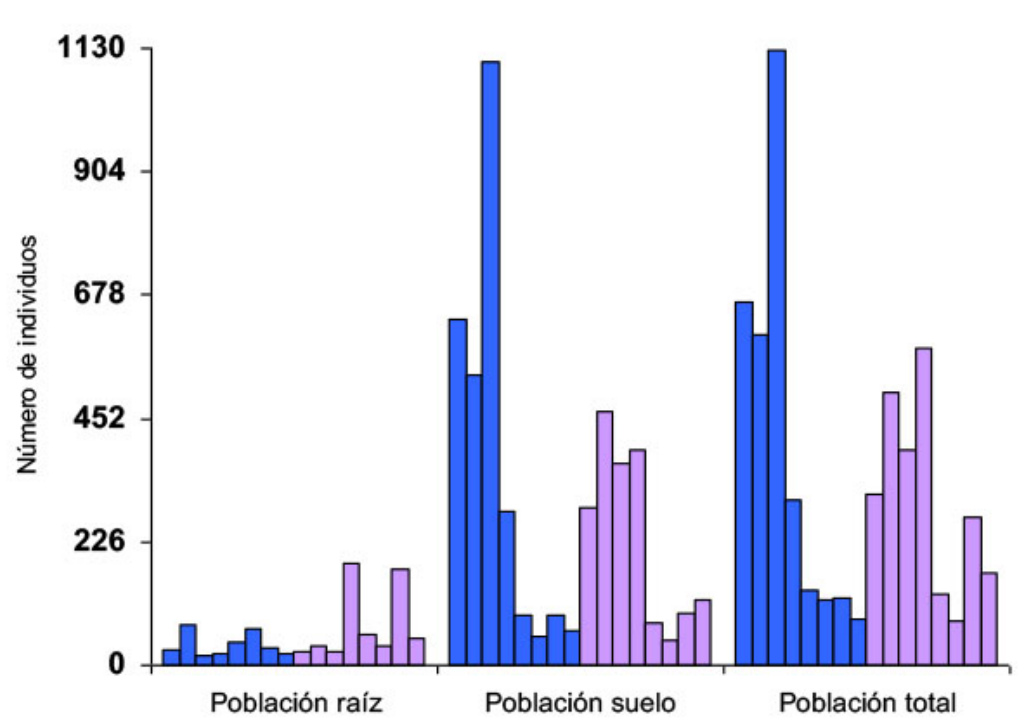

Figura 1. Población de nematodos fitopatógenos cuantificados en raíz y suelo cultivados con plantas de plátano (Musa AAB cv. Hartón) de alto y bajo vigor, en el sur del lago de Maracaibo, Venezuela. 2018.

Columnas acompañadas por letras distintas señalan diferencias estadísticas entre lotes de plantas de plátano $(\mathrm{p}<0,05)$. Cada barra representa el número de lotes de alto y bajo vigor de plantas de plátano de cada unidad de producción.

Figure 1. Population of phytopathogenic nematodes quantified in root and soil grown with high and low-vigor plantain (Musa AAB cv. Hartón) plants, in the south of lake Maracaibo, Venezuela. 2018.

Columns accompanied by different letters point to statistical differences between lots of plantain plants ( $<<0.05)$. Each bar represents the number of high and low-vigor plantain plants lots in each production unit.

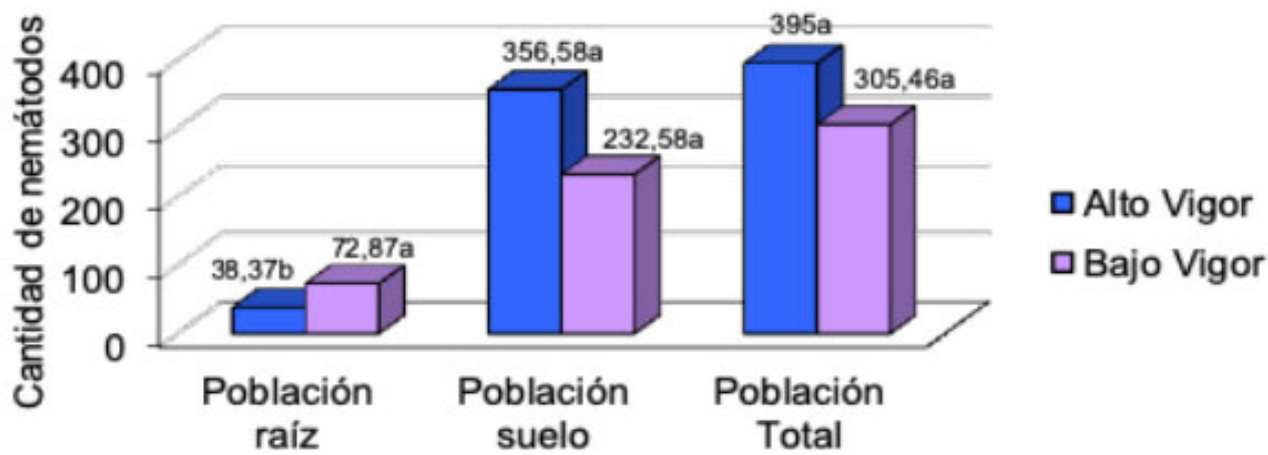

Figura 2. Población de nematodos fitopatógenos cuantificados en lotes de plantas de plátano (Musa AAB cv. Hartón) de alto y bajo vigor en el sur del lago de Maracaibo, Venezuela. 2018.

Columnas acompañadas por letras distintas señalan diferencias estadísticas entre lotes de plantas de plátano $(\mathrm{p}<0,05)$. Cada barra representa el total de lotes de alto y bajo vigor de plantas de plátano.

Figure 2. Population of phytopathogenic nematodes quantified in batches of high and low-vigor plantain (Musa AAB cv. Hartón) plants, in the south of lake Maracaibo, Venezuela. 2018.

Columns accompanied by different letters point to statistical differences between plantain plants lots $(\mathrm{p}<0,05)$. Each bar represents the total of high and low-vigor plantain plants lots. 
suelo de lotes de plantas de plátano con alto vigor con respecto a los de bajo vigor. Mientras que, en las muestras de raíz, a pesar de que la población acumulada fue la más baja, los resultados indicaron diferencias estadísticamente significativas entre lotes de plantas de diferente vigor, en el caso de bajo vigor presentó el 65,60 \% de la población total, mientras que los de alto solo el $34,39 \%$.

La conformación poblacional de las muestras procesadas, independientemente del lote de vigor de plantas de plátano y del tipo de muestra estudiada (raíz y suelo), se encuentra representada en la Figura 3, donde fueron agrupados doce géneros taxonómicos distintos.

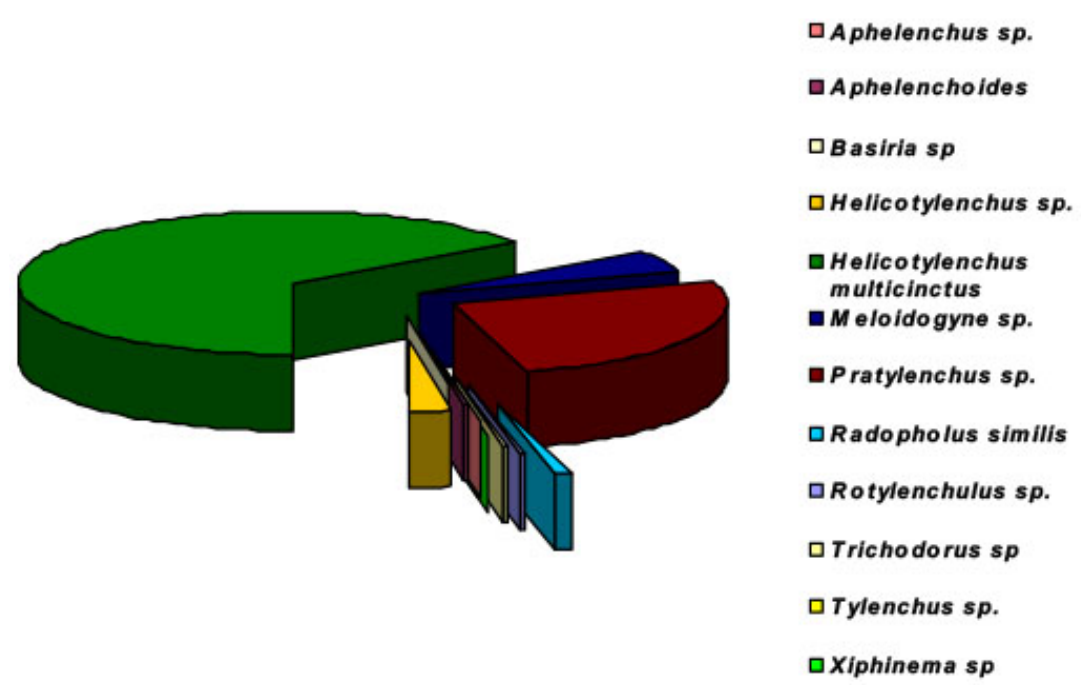

Figura 3. Géneros taxonómicos que conforman las poblaciones de nematodos fitopatógenos cuantificadas en plantaciones de plátano (Musa AAB cv. Hartón) del sur del lago de Maracaibo, Venezuela. 2018.

Figure 3. Taxonomic genera that make up quantified phytopathogenic nematode populations on plantain (Musa AAB cv. Hartón) plantations in the south of lake Maracaibo, Venezuela. 2018.

Adicionalmente, se observó que en promedio los lotes de alto vigor presentaron mayor cantidad de nematodos fitopatógenos que los de bajo vigor (Cuadro 3), indiferente del tipo de muestra o ubicación. Los nematodos de mayor importancia fitosanitaria en el lote de alto vigor, fueron Helicotylenchus multicinctus, Radopholus similis y Meloidogyne sp.

\section{Discusión}

Los resultados de determinación de vigor de plantas de plátano de diferentes fincas fueron similares a los encontrados por González-Pedraza et al. (2014), en una finca con características de suelos similares, cultivada con plátano Hartón, ubicada en el municipio Francisco Javier Pulgar del estado Zulia; con mayor número de manos por racimo, circunferencia del pseudotallo de la planta madre y altura del hijo de sucesión mayor en lotes de alto vigor en comparación con los lotes de bajo vigor. 
Cuadro 3. Cuantificación poblacional por género de nemátodo fitopatógeno identificado en el tipo de muestra y lotes de plantas de plátano (Musa AAB cv. Hartón) con diferente vigor en el sur del lago de Maracaibo, Venezuela. 2018.

Table 3. Population quantification by genus of phytopathogenic nematode identified in the sample type and batches of plantain (Musa AAB cv. Hartón) plants with different vigor in the south of lake Maracaibo, Venezuela. 2018.

\begin{tabular}{|c|c|c|c|c|c|c|c|c|}
\hline \multirow{2}{*}{$\begin{array}{l}\text { Género de nematodo } \\
\text { fitopatógeno }\end{array}$} & \multicolumn{5}{|c|}{ Tipo de muestra (ubicación) } & \multicolumn{3}{|c|}{ Lotes de vigor de plantas } \\
\hline & Raíz & $\begin{array}{l}\text { Total de } \\
\text { raíz }\end{array}$ & Suelo & $\begin{array}{l}\text { Total de } \\
\text { suelo }\end{array}$ & $\begin{array}{l}\text { Alto } \\
\text { vigor }\end{array}$ & $\begin{array}{l}\text { Total de } \\
\text { alto vigor }\end{array}$ & $\begin{array}{l}\text { Bajo } \\
\text { vigor }\end{array}$ & $\begin{array}{c}\text { Total de } \\
\text { bajo vigor }\end{array}$ \\
\hline Aphelenchus sp. & $0,04 \mathrm{a}$ & 2 & $0,39 a$ & 19 & $0,37 \mathrm{a}$ & 18 & $0,062 \mathrm{a}$ & 3 \\
\hline Aphelenchoides & $0,02 \mathrm{a}$ & 0 & $0,31 \mathrm{a}$ & 15 & $0,27 \mathrm{a}$ & 13 & $0,06 \mathrm{a}$ & 3 \\
\hline Basiria sp. & $0,02 \mathrm{a}$ & 1 & $0 \mathrm{a}$ & 0 & $0,02 \mathrm{a}$ & 1 & $0 \mathrm{a}$ & 0 \\
\hline Helicotylenchus sp. & $6,18 \mathrm{a}$ & 297 & $3,08 \mathrm{a}$ & 148 & $4,08 \mathrm{a}$ & 196 & $5,18 \mathrm{a}$ & 249 \\
\hline Helicotylenchus multicinctus & $26,85 b$ & 1289 & $200,65 a$ & 9631 & $148,52 \mathrm{a}$ & 7129 & $78,97 b$ & 3791 \\
\hline Meloidogyne sp. & $1,52 b$ & 73 & $16,18 \mathrm{a}$ & 777 & $12,20 \mathrm{a}$ & 586 & $5,50 \mathrm{a}$ & 264 \\
\hline Pratylenchus sp. & $18,52 b$ & 889 & $70,50 \mathrm{a}$ & 3384 & $27,39 b$ & 1315 & $61,62 \mathrm{a}$ & 2958 \\
\hline Radopholus similis & $1,54 \mathrm{a}$ & 74 & $2,47 \mathrm{a}$ & 119 & $3,48 \mathrm{a}$ & 167 & $0,54 b$ & 26 \\
\hline Rotylenchulus sp. & $0,77 \mathrm{a}$ & 37 & $0,33 \mathrm{a}$ & 16 & $0,46 \mathrm{a}$ & 22 & $0,64 \mathrm{a}$ & 31 \\
\hline Trichodorus sp. & $0 \mathrm{a}$ & 0 & $0,39 \mathrm{a}$ & 19 & $0,35 \mathrm{a}$ & 17 & $0,04 \mathrm{a}$ & 2 \\
\hline Tylenchus sp. & $0,17 \mathrm{a}$ & 8 & $0,06 \mathrm{a}$ & 3 & $0,17 \mathrm{a}$ & 8 & $0,06 \mathrm{a}$ & 3 \\
\hline Xiphinema sp. & $0 \mathrm{a}$ & 0 & $0,19 \mathrm{a}$ & 9 & $0,17 \mathrm{a}$ & 8 & $0,02 \mathrm{a}$ & 1 \\
\hline
\end{tabular}

Valores promedios seguidos por letras minúsculas diferentes entre las columnas indican diferencias significativas $(\mathrm{p}<0,05)$ según prueba Tukey entre tipos de muestras o ubicación en los diferentes géneros y lotes de vigor de plantas de plátano / Average values followed by different lowercase letters between the columns indicate significant differences $(p<0,05)$ according to the Tukey test between sample types or location in the different genera and vigor lots of plantain plants.

Los valores promedios para la circunferencia de la planta madre $(\mathrm{cm})$ fueron relativamente altos, en comparación con los datos reportados $(55,9 \mathrm{~cm})$ por Delgado et al. (2008), en un trabajo realizado en la región central del estado Barinas bajo condiciones de suelo y clima muy similares a las de este estudio, en plantaciones de plátano (Musa AAB cv. subgrupo plátano Hartón) con densidades de siembra de 1111 plantas ha-1.

Se observó que la cantidad de nematodos varió según el tipo de muestra procesada, hubo mayor población en el suelo (14 140 individuos, equivalente al $84 \%$ de la población total) que en la raíz (2670 individuos, equivalente al 15 $\%$ de la población total). Estas diferencias fueron estadísticamente significativas. La alta presencia de este grupo de fitopatógenos en el suelo, independientemente del tipo de condición de vigor, se asoció directamente al ecosistema primario como habitat, lo cual hace referencia al cultivo, a la rizósfera del mismo, a los niveles de parasistismo que pueden presentar los géneros encontrados en la poblacion cuantificada (Agrios, 2005; Montiel et al., 1997) y a los resultados poblacionales en las muestras de raíz. No hubo respuesta significativa entre lotes de vigor, pero se observó un incremento poblacional en suelo de 23,04 \% entre las plantas de alto vigor, en comparación con las de bajo. Esta respuesta se pudo originar probablemente al incremento de actividad microbiana como resultado en los cambios de concentración de los minerales presentes, asociado al manejo y a la utilización de productos biológicos (Coto, 2007), lo que le permitió establecer las condiciones óptimas para el crecimiento y multiplicación poblacional en este medio (Araya et al., 2011). Es posible suponer que al ser el tejido radical el huesped y constituir la fuente de alimento para los parasitos la población, se mueve en mayor presencia hacia la raíz, lo que reduciría la población 
presente en suelo tal como sucedió en los lotes de bajo vigor. Esto explicaría la relación entre la alta población de nematodos en las plantas de bajo vigor y los resultados bajo índices en los parámetros biométricos obtenidos para este criterio. Al respecto, se explicó que la presencia de estos fitopatógenos en la raíz de banana puede conducir al retraso del crecimiento de las plantas, al alargamiento del ciclo vegetativo, la reducción del tamaño de la planta y el peso del racimo (Gowen et al., 2005). Asimismo, el parasitismo de nematodos sobre raíces de banana, así como en muchos cultivos tropicales, se caracteriza por infestaciones simultaneas de varias especies; este comportamiento fue observado en la identificación de la nematofauna parasítica asociada al cultivo, encontrándose ocho géneros distintos en raíz y 10 en suelo, el leve incremento de estos resultados se atribuyó a la diversidad edáfica propia de cada agroecosistema (Gowen et al., 2005). Por orden de cuantía en las muestras se tipificaron los géneros: Helicotylenchus, Pratylenchus, Radopholus, Meloidogyne, Rotylenchulus, Tylenchus, Aphelenchus, Basiria, Trichodorus, Xiphinema y Aphelenchoides.

Helicotylenchus multicinctus fue el nemátodo más frecuente en el estudio, tanto en muestras de suelo como de raíz, al respecto, Mcsorley \& Gallaher (1995), explicaron que esta especie podría superar numéricamente a $R$. similis en localidades donde las especies coexisten y en áreas subtropicales donde el nemátodo barrenador se presenta. En Venezuela, la presencia de H. multicinctus asociado al cultivo ha sido reportada por Crozzoli (2009) en sus diagnósticos de banano y plátano, quien lo señala como la especie más abundante, en el estudio fueron 10 920 individuos encontrados. La mayor presencia en las muestras de suelo que en las de raíz es el resultado del hábitat parasítica del espécimen, $H$. multicinctus se indica como una de las especies más agresiva, causa daños al cultivo de plátano con la reducción en el peso del racimo y el tiempo de vida útil de las plantaciones (Torrado-Jaime \& Castaño-Zapata, 2009). Sin embargo, no existe ningún estudio en cual se determine el umbral de daño de los nematodos fitoparasíticos asociados a banano y plátano en Venezuela (Crozzoli, 2009).

Otros estudios en la zona sur del Lago de Maracaibo, han reportado en plantas de plátano los géneros: Helicotylenchus, Radopholus, Meloidogyne y Aphelenchoides, en cuyos casos las poblaciones fueron mayores en muestras de raíz que en las de suelo (Labarca et al., 2011). Aunque las poblaciones no fueron similares a las del presente estudio, su identificación en las muestras indicaron que la diversidad de fitonematodos prevalece en la zona, a pesar del manejo y del clima.

Se destaca que el género Radopholus se ha encontrado en muy bajas proporciones y solo en muestras de raíces en el sur del lago de Maracaibo (Abou Assi et al., 2009; Montiel et al., 1997), lo que coincide con los resultados obtenidos en este estudio. Aunque contrariamente, Labarca et al. (2011) mostraron una población máxima de 780 nematodos por $10 \mathrm{~g}$ de raíces en el sector Caño muerto del sur del lago de Maracaibo, probablemente esta fluctuación se pudo asociar a las condiciones edafoclimáticas de este sector en particular, las condiciones de drenaje en las plantaciones y la época del año de este grupo de estudio ya que estos factores pueden incidir en los aumentos o descensos de las poblaciones microbianas edáficas (Agrios, 2005).

Meloidogyne fue otro de los géneros identificados con una población total de 850 nematodos, mayor a los encontrados por Labarca et al. (2011), con 502 nematodos en muestras de raíces y suelos en el sector Caño muerto. Este género ha sido vinculado en banano como el agente causal de lesiones severas en raíces, deficiencias en el crecimiento, reducción en el peso del racimo y pérdida en el rendimiento (Adriano-Anaya et al. 2008).

Aunque en el estudio no se asoció la cantidad de un género en raíz por lote de planta, se observó una tendencia de alta presencia de los géneros Helicotylenchus, Pratylenchus y Meloidogyne en ambos lotes y se destaca el incremento de las poblaciones totales de Pratylenchus en los lotes de bajo vigor, por lo que se podría suponer que el resultado del desempeño de vigor de las plantas estuvo asociado a la presencia de los géneros encontrados y la compleja actividad patogénica entre ellos para ocasionar lesiones importantes en las raíces de las plantas. La presencia de este nemátodo fitopatógeno fue reportada por Labarca et al. (2011) en el sector Caño muerto 470 nematodos $10 \mathrm{~g}^{-1}$ raíces del género Pratylenchus. 


\section{Conclusiones}

Se identificaron doce géneros taxonómicos de nematodos, lo cual indica una alta diversidad de la nematofauna presente tanto en los suelos estudiados como en raíces del cultivo de plátano.

La mayor población total de individuos se encontró en las muestras de suelos, los géneros de fitonematodos identificados fueron: Helicotylenchus multicinctus, Pratylenchus sp. y Meloidogyne sp.

En lotes con plátano de alto vigor se identificaron los géneros: Helicotylenchus multicinctus, y Radopholus similis, y en lotes de bajo vigor: Pratylenchus sp.

\section{Referencias}

Adriano-Anaya, M., Herrera-López, D., Albores-Flores, V., Salvador- Figueroa, M., \& Velasco-Zebadua, M. (2008). Nematodos endorrizosféricos del banano (Musa AAA. subgrupo Cavendish) clon «Grande Naine» en el Soconusco, Chiapas, México. Revista Mexicana de Fitopatología, 26(2),147-152. https://www.redalyc.org/articulo.oa?id= 61226207

Agrios, G. (2005). Fitopatología. 2da ed. Editorial Limusa.

Araya, M., Serrano, E., \& Vargas, A. (2011). Relación entre el contenido de nutrientes en suelo y raíces de banano (Musa AAA) con el peso de raíces y número de nematodos. Fitosanidad, 15(3), 163-177. https://www.redalyc.org/ pdf/2091/209122682006.pdf

Arias, F. (2006). El proyecto de Investigación. Introducción a la metodología científica. $5^{\mathrm{a}}$ ed. Editorial Episteme.

Abou Assi, K., Guillén, J., Labarca, J., Casassa, A., Paredes, C., Casanova, M., \& Sandoval, L. (2009). Nematodos fitoparasíticos asociados al cultivo del plátano (Musa AAB. cv. Hartón) en bosque seco tropical. Revista Científica UDO Agrícola, 9(1),199-207. http://udoagricola.orgfree.com/V9N1UDOAg/V9N1Assi199.htm

Badri, D., \& Vivanco, J. (2009). Regulation and function of root exudates. Plant, Cell \& Environment, 32(6), 666-681. https:// doi.org/10.1111/j.1365-3040.2008.01926.x

Bautista, L., Bolaños, M., Massae, A., \& Villegas, B. (2015). Respuesta de fitonematodos de plátano Musa AAB Simmonds a estrategias de manejo integrado del suelo y nutrición. Revista Luna Azul, 40, 69-84. https://doi.org/10.17151/ luaz.2015.40.6

Belalcázar, S. (1991). El cultivo del plátano (Musa AAB Simmonds) en el trópico. Manual de asistencia técnica $\mathrm{N}^{\circ} 50$. Instituto Colombiano Agropecuario. http://hdl.handle.net/20.500.12324/12434

Coto, G. (2007). Conceptos introductorios a la fitopatología. Editorial Universidad Estatal a Distancia.

Crozzoli, R. (2009). Nematodes of tropical fruit crops in Venezuela. In A. Ciancio, \& K. Mukerji, (Eds.), Integrated management of fruit crops and forest nematodes (pp. 63-84). Springer.

Crozzoli, R. (2002). Especies de nematodos fitoparasíticos en Venezuela. Interciencia, 27(7), 354-364.

Delgado, E., Gómez, N., González, O., \& Marín, C. (2008). Evaluación a nivel de finca del efecto de la alta densidad de siembra en plátano (Musa AAB cv. Subgrupo plátano Hartón), municipio Obispo, Barinas, Venezuela. Revista de la Facultad de Agronomía (LUZ), 25(4), 603-616. 
Dropkin, V. (1989). Introduction to plant nematology. $2^{\text {nd }}$ ed. John Wiley \& Sons.

Food and Agriculture Organization. (2004). La economía mundial del banano 1985-2002. http://www.fao.org/3/y5102s/y5102 s00.htm\#Contents.

González-Pedraza, A., Atencio, J., Cubillán, K., Almendrales, R., Ramírez, L., \& Barrios, O. (2014). Actividad microbiana en suelos cultivados con plátano (Musa AAB subgrupo plátano cv. 1 Hartón) con diferente vigor de plantas. Revista de la Facultad de Agronomía (LUZ), Supl. 1, 526-538. https://www.researchgate.net/publication/289083887_Microbial_ activity_in_soils_cultivated_with_plantain_Musa_AAB_plantain_subgroup_cv_Harton_with_different_vigor_of_ plants

Gómez, C., Vera, J., Romero, J., Timaure, R., Valery, A., \& Caña, J. (2016). Evaluación de prácticas de manejo del racimo de plátano "Hartón" (Musa AAB) en el municipio Colón, estado Zulia. Revista Producción Agropecuaria, 5(1), 8-13. http://www.investigacion.unesur.edu.ve/index.php/rpa/article/view/73

Gowen, R., \& Quénéhervé, P. (1990). Nematode parasites of bananas, plantains and Abaca. In M. Luc, R. Sikora, \& J. Bridge, (Eds.), Plant parasitic nematodes in subtropical and tropical agriculture (pp. 431-460). CAB International.

Gowen, S., Quénéhervé, P. \& Fogain, R. (2005). Nematode parasites of bananas and plantains. In M. Luc, R. A. Sikora, \& J. Bridge, (Eds.), Plant parasitic nematodes in subtropical and tropical agriculture (2nd ed., pp. 611-644). CAB International.

Hernández, Y., Marín, M., \& García, J. (2007). Respuesta en el rendimiento del plátano (Musa AAB cv. Hartón) en función de la nutrición mineral y su ciclo fenológico. Parte I. Crecimiento y producción. Revista de la Facultad de Agronomía (LUZ), 24(4), 607-626.

Jacob, J., \& Van-Bezooijen, J. (1971). A manual for practical work in nematology. Agricultural University of Wageningen.

Kijerwski, J., Colina, L., Steegmayer, P., \& Patiño, H. (1976). Estudio agrológico semidetallado, zona Sur del Lago, río Mucujepe-río Escalante. Ministerio del Ambiente y de los Recursos Naturales Renovables.

Labarca, J., Casassa, A., Pineda, M., Ulacio, D., Casanova, M., \& Sandoval, L. (2011). Diagnóstico de nematodos fitoparásitos en plátano (Musa AAB) cv. Hartón en el Sur del Lago de Maracaibo. Revista de la Facultad de Agronomía (LUZ), 28(1), 213-227. https://produccioncientificaluz.org/index.php/agronomia/article/view/26995

Martínez, G. (2009). Situación nacional de las musáceas: Breve análisis. Producción Agropecuaria, 2(1), 31-44. http:// investigacion.unesur.edu.ve/index.php/rpa/article/view/40

Mcsorley, R., \& Gallaher, R. (1995). Effect of yard waste compost on plant-parasitic nematode densities in vegetable crops. Journal of Nematology, 27(4S), 545-549. https://www.ncbi.nlm.nih.gov/pmc/articles/PMC2619647/pdf/545.pdf

Montiel, A., Sosa, L., Medrano, C., \& Romero, D. (1997). Nematodos fitoparásitos en plantaciones de plátano (Musa AAB) de la margen izquierda del río Chama, Estado Zulia, Venezuela. Revista de la Facultad de Agronomía (LUZ), 14(2), 245-251.

Nava, C. (1997). El plátano. Su cultivo en Venezuela. Ediciones Astro Data.

Peña, C., Dayakar, B., \& Loyola, V. (2012). Plant root secretions and their interactions with neighbors. In J. Vivanco, J., \& F. Baluŝka, (Eds.), Secretions and exudates in biological systems, Signaling and communication in plants (vol 12, pp. 1-26). Springer. https://doi.org/10.1007/978-3-642-23047-9_1 
Rodríguez, V., \& Rodríguez, O. (1998). Biometría de la cepa de plátano (Musa AAB subgrupo plátano cv. Hartón) en plantas con rendimientos superiores a 18 kilogramos por racimo, en Venezuela. Revista de la Facultad de Agronomía (LUZ), 15(5), 439-445. https://produccioncientificaluz.org/index.php/agronomia/article/view/26218

Rosales, F., Pocasangre, L., Trejos, J., Serrano, E., \& Peña, W. (2008). Guía de diagnóstico de la calidad y salud de suelos. Bioversity International. https://www.bioversityinternational.org/fileadmin/_migrated/uploads/tx_news/Guia_de_ diagnostico_de_la_calidad_y_salud_de_suelos_bananeros_1375.pdf

Soto, M. (2008). Banano: técnicas de producción, manejo, postcosecha y comercialización. $3^{\text {era }}$ ed. Litografía e Imprenta LIL.

Torrado-Jaime, M., \& Castaño-Zapata, J. (2009). Incidencia de nematodos en plátano en distintos estados fenológicos. Revista Agronomía Colombiana, 27(2), 237-244. https://revistas.unal.edu.co/index.php/agrocol/article/view/11199/37783

Torres-Guerrero, C., Etchevers, J., Fuentes-Ponce, M., Govaerts, B., De León-González, F., \& Herrera, J. (2013). Influencia de las raíces sobre la agregación del suelo. Terra Latinoamericana, 31(1), 71-84. https://www.redalyc.org/ pdf/573/57327411007.pdf 